\title{
A Gated Four Probe Technique for Field Effect Measurements on Disordered Organic Semiconductors
}

\author{
Elizabeth von Hauff, Nicolas Spethmann, and Jürgen Parisi \\ Institute of Physics, Department for Energy and Semiconductor Research, \\ Carl von Ossietzky University of Oldenburg, D-26111 Oldenburg, Germany \\ Reprint requests to E. v.H.; E-mail: elizabeth.v.hauff@uni-oldenburg.de
}

Z. Naturforsch. 63a, 591 - 595 (2008); received May 15, 2008

\begin{abstract}
A gated four probe measurement technique to isolate contact resistances in field effect measurements on disordered organic semiconductors was investigated. Organic field effect transistors (OFETs) were prepared with two additional electrodes in the contact geometry protruding into the source-drain channel to monitor the variation in potential across the channel. Two high impedance electrometers were used to determine the potential at the contacts. This technique allows to directly determine the magnitude of the parasitic contact resistances between metal contact and organic semiconductor from the drop in potential at the contact regions. The effects of contact resistances, which can falsify measurements on bulk transport parameters such as the field effect mobility, can be then eliminated during material characterization. Additionally, the temperature and electric field dependence of the contact resistances offers valuable information about the charge injection and extraction processes between metal and organic semiconductor. The effects of the four probe geometry, specifically the effect of the channel electrodes on the current-voltage characteristics, of hole transport in a polythiophene (P3HT) OFET with Au contacts were investigated and found not to influence device performance, except at currents $\ll 1 \mathrm{nA}$. The IV characteristics were shown to follow the expected FET behaviour. From the variation in potential along the channel it was found that contact resistances at the source contact (charge injecting contact) are minimal while contact resistances at the drain contact (charge extracting contact) are significant, resulting in a much lower effective sourcedrain voltage than that applied to the device.
\end{abstract}

Key words: Contact Resistances; Organic Field Effect Transistor.

\section{Introduction}

The organic field effect transistor (OFET) is a useful tool for investigating charge carrier transport properties in organic semiconductors. Solution-deposited organic semiconductors have an amorphous nature; charge carriers are only delocalized over molecular segments, and charge transport through the bulk semiconductor film must occur via temperature and/or electric field-activated hopping between charge transport sites [1].

Investigations of the temperature and field dependence of the field effect mobility offer valuable information about charge transport in organic semiconductors for a variety of applications in organic electronics. One particular advantage of field effect mobility measurements is that the electron and hole mobilities can be investigated independently in the same device simply by reversing the polarity of the applied source-drain and gate voltages. This is very useful for investigations of ambipolar transport in organic films or in compositions of more than one type of organic semiconductor, e.g. in polymer solar cells, in which the absorber layer consists of a conducting polymer combined with a fullerene derivative to create an electron donor-acceptor system [2-4]. Field effect measurements can additionally be used as a simple method of screening the effect of processing steps on charge transport in the organic semiconducting layers, as changes in the field effect mobility indicate changes in the film quality [5].

OFETs use the thin film transistor geometry shown in Figure 1. Quite often for material characterization purposes a simplified OFET structure is used: a highly doped silicon wafer serves as the gate electrode. A gate insulator, such as thermally grown $\mathrm{SiO}_{2}$, electrically insulates the semiconductor layer from the gate electrode. Either the source and drain contacts are deposited onto the gate insulator (bottom contact) and the semiconducting layer is applied as the final step, 


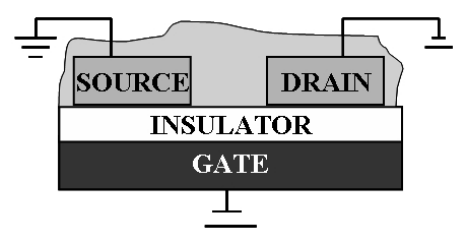

Fig. 1. Cross-section of an OFET. The gate electrode is electrically insulated from the rest of the device by the gate insulator. Source and drain contacts are patterned onto the gate insulator (bottom contact) or onto the semiconductor layer (top contact).

or the contacts are deposited onto the semiconductor layer (top contact). Device processing for bottom contact devices is more practical and results in less stress on the organic layer, however, parasitic contact effects which have a negative effect on device behaviour are more pronounced in bottom contact OFETs than top contact OFETs $[6,7]$.

OFETs operate in accumulation mode, i. e., current is made up of majority carriers and the low conductivity of the organic material controls the on/off behaviour of the device. Applying a positive/negative voltage to the gate electrode $\left(V_{\mathrm{G}}\right)$ results in an accumulation of electrons/holes in the source-drain channel along the gate insulator-semiconductor interface, and applying a positive/negative source-drain voltage $\left(V_{\mathrm{DS}}\right)$ results in an electron/a hole current $\left(I_{\mathrm{DS}}\right)$ through the channel. The current increases linearly at low $V_{\mathrm{DS}}$, and saturates at higher $V_{\mathrm{DS}}$. The width $(W)$ and separation of the contacts (channel length $L$ ) determines the magnitude of current in the channel. The field effect mobility $\left(\mu_{\mathrm{FE}}\right)$ can be determined from the IV characteristics. Due to the field dependence of the transport, $\mu_{\mathrm{FE}}$ is typically determined in the linear regime of the IV characteristics for organic semiconductors [8].

Parasitic contact resistances are known to significantly influence experimentally determined values of the field effect mobility in contact limited devices $[9,10]$. A drop in the source-drain voltage in the contact region leads to a lower effective source-drain voltage across the channel, leading to a lower channel current, thus resulting in a value for the experimentally determined field effect mobility that is lower than the actual value in the material. The physics at the metal-organic semiconductor interface are poorly understood, and cannot be accounted for from the work function of the metal contact and the energetics of the semiconductor material alone. In the literature this has been attributed to such effects as interface dipoles [11, 12] and Fermi level pinning [13].
The effects of contact resistances in OFETs have already been investigated and accounted for in field effect measurements in the literature using several methods. The most common is the transmission line method (TLM) in which a series of samples is fabricated under the same conditions, and the channel length is varied between the samples. The total device resistance increases with increasing channel length, but the contact resistance remains the same between samples and can be determined from the current-voltage characteristics of the device series $[14,15]$. The major disadvantage associated with the TLM, aside from being relatively time-consuming and inaccurate due to the error associated with the large number of samples measured, there is no way to differentiate between source contact resistances and drain contact resistances. This is possible using Kelvin probe force microscopy [16]. For investigations relating to material characterization, however, a direct method of determining the magnitude and effects of the source and drain contact resistances during the field effect measurement is desirable.

One practical possibility is to perform a gated four probe measurement by patterning thin, highly ohmic electrodes that protrude into the source-drain channel to monitor the potential drop along the channel and relate this to the potential drop in the contact regions. The externally applied source-drain voltage is known, and via the channel electrodes, the actual source-drain voltage, after losses due to contact resistances, can be determined and corrected for. The gated four probe geometry has already been investigated for pentacene OFETs [17]. The channel probes were patterned onto the semiconductor layer after device production. It was found that for a suitable device geometry the fingers do not influence the potential in the channel.

In this study a four probe geometry for field effect measurements on polythiophene (P3HT) OFETs was investigated. To make the material characterization more practical and reduce damage to the semiconducting layer, the entire contact geometry, i. e. the source and drain contacts and the channel electrodes, was patterned directly onto the gate insulator. The P3HT layer was then applied over the complete contact structure. Hole transport in the polymer was investigated, and it was found from the output characteristics that the channel contacts do not influence the FET behaviour of the device. The field effect mobilities determined from P3HT OFETs without correction for the effects of contact resistances were in the low $10^{-3} \mathrm{Vs} / \mathrm{cm}^{2}$ range. The variation in potential along the channel was 
examined and the magnitude of the contact resistances in the device was investigated. After considering the effects of contact resistances leading to a significant potential drop along the channel, $\mu_{\mathrm{FE}}$ was found to be in the higher $10^{-3} \mathrm{Vs} / \mathrm{cm}^{2}$ range.

\section{Experimental}

Two OFET structures were used in this study. The first structure was the gated four probe geometry shown in Figure 2. Highly doped silicon wafers with $100 \mathrm{~nm}$ of thermally grown $\mathrm{SiO}_{2}$ were purchased from MicroFAB. The contact material used was Au. The source and drain electrodes were fabricated in a rectangular geometry on the substrate with a channel width of $1400 \mu \mathrm{m}$ and a channel length of $50 \mu \mathrm{m}$. Two thin electrodes ( $W \sim 6 \mu \mathrm{m}, L=400 \mu \mathrm{m}$ ) were applied inside the channel. All structures were defined using optical photolithography.

The second structure was used for comparison to the four probe geometry, and was an interdigitated drainsource geometry used to achieve a high $W / L$ ratio (Fig. 3). The channel length and channel width were $L=10 \mu \mathrm{m}$ and $W=10 \mathrm{~mm}$, respectively. The contact metal was Au. The patterned samples were purchased from the Fraunhofer Institute for Photonic Microsystems.

After performing photolithography to pattern the contacts for the four point structures, the substrates were cleaned in a solution of $\mathrm{NH}_{4} \mathrm{OH} / \mathrm{H}_{2} \mathrm{O}_{2}(30 \%)$ to remove any remainder of organic photo resist [18]. Both the four probe and interdigitated structures were exposed to oxygen plasma for $10 \mathrm{~min}$ before applying the organic layer.

The organic semiconductor was applied to the substrate as the final step. Organic layers were spin-coated at approximately $1300 \mathrm{rpm}$. The concentration of the

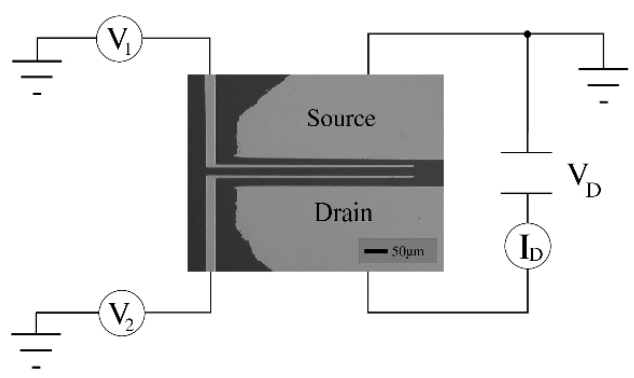

Fig. 2. Four probe geometry. Two thin fingers are brought into the conductive channel to determine the variation in potential along the source-drain channel. The measurement scheme for the device is also shown.

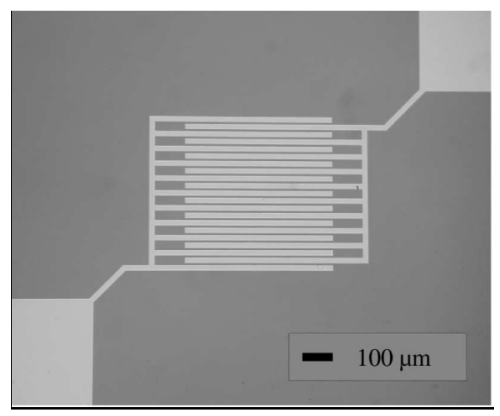

Fig. 3. Interdigitated OFET structure. $L=10 \mu \mathrm{m}$ and $W=$ $10 \mathrm{~mm}$. Source and drain were contacted for measurement via the larger contact structures in the corners of the figure.

solution was $2 \mathrm{mg}$ P3HT per ml chloroform. All processing steps for fabricating the organic semiconducting layer were done in a glove box under nitrogen atmosphere.

The prepared samples were transferred into a cryostat for measurement. The samples were electrically characterized in the dark under a pressure of $10^{-6}$ mbar.

Current-voltage characteristics were acquired with a Source Measure Unit (Keithley 236). The gate voltage was applied to the common gate with a voltage source (Keithley 2400). For the gated four probe technique, the measurement of the potentials at the channel electrodes was performed independently using two high impedance $(\sim 200 \mathrm{~T} \Omega)$ electrometers (Keithley 6517) to minimize the current in the electrodes. In Fig. 2 the measurement scheme for the four probe structure is sketched. A LabView program was used for automated measurements.

\section{Results}

The output characteristics of P3HT OFETs fabricated on the four probe structures were investigated. It was found that the IV characteristics of such devices followed the expected electrical behaviour of a field effect transistor: $I_{\mathrm{DS}}$ increases linearly for low $V_{\mathrm{DS}}$ and levels off at higher $V_{\mathrm{DS}}$. Figure 4 shows the FET characteristics of the device on the left-hand side. On the right-hand side, for comparison, the FET characteristics of a P3HT OFET fabricated using a two point interdigitated contact structure are shown. The field effect mobility was determined from the linear regime of the FET characteristics for both device structures without considering the effects of contact resistances and was found to be in the low $10^{-3} \mathrm{Vs} / \mathrm{cm}^{2}$ range. It should 


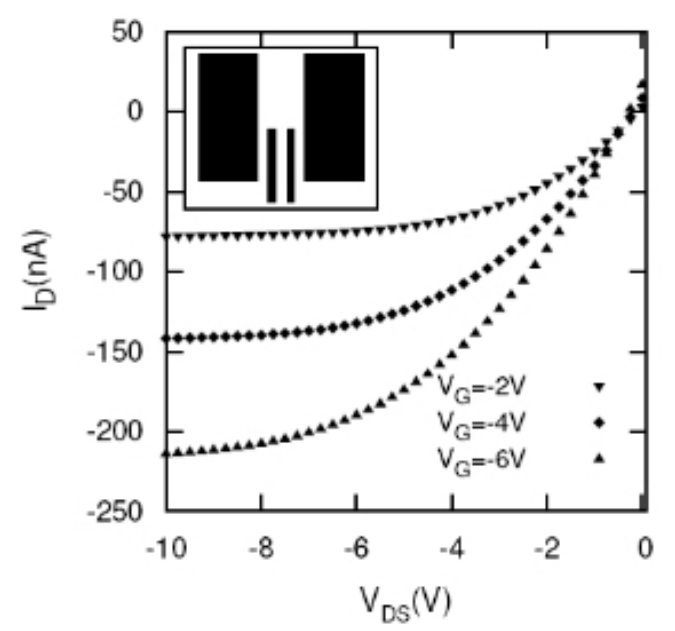

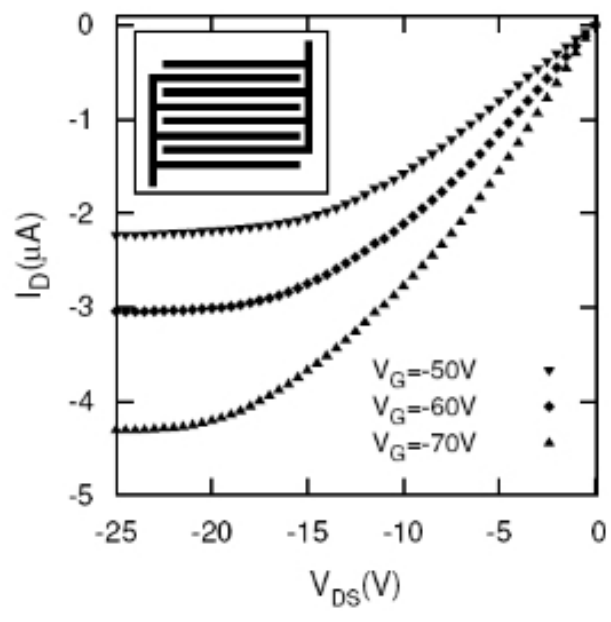

Source

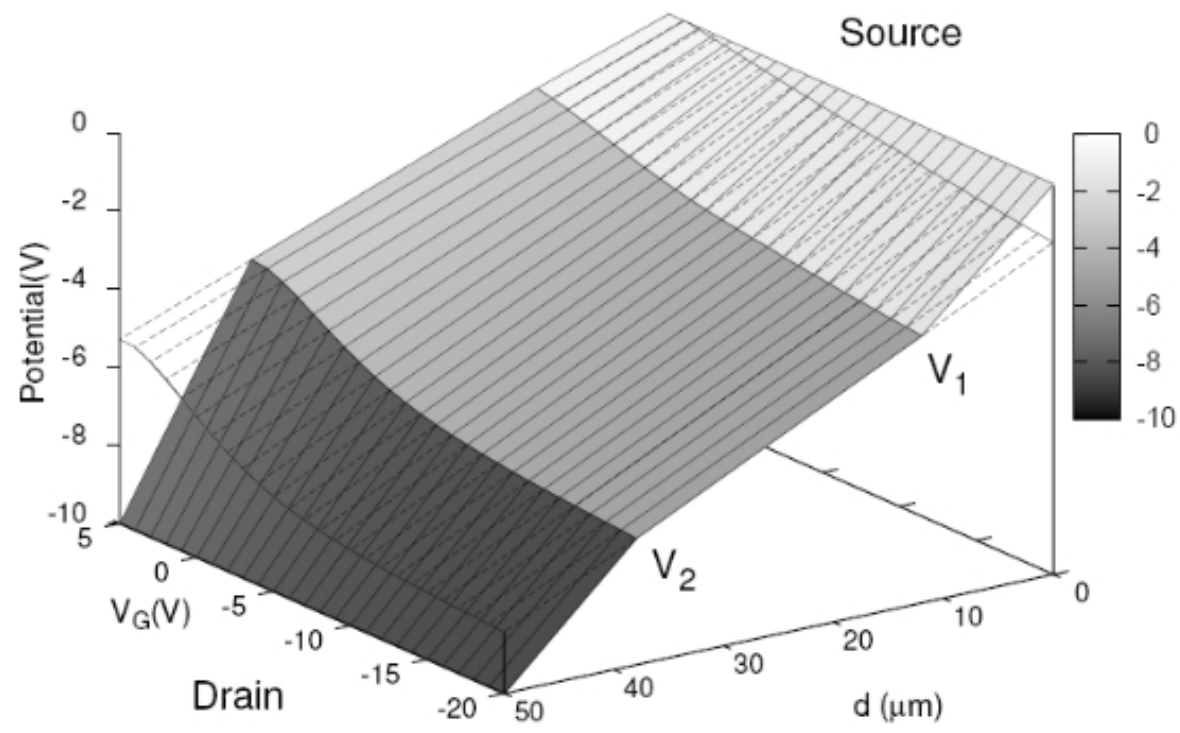

Fig. 4. Currentvoltage characteristics of P3HT OFETs fabricated on a four probe geometry (left) and on an interdigitated contact geometry (right).

Fig. 5. Variation in the potential along the sourcedrain channel in dependence on the source-drain voltage and gate voltage. $V_{1}$ and $V_{2}$ are the potentials measured at the position of the channel electrodes.

be note that no optimization steps were undertaken to improve the mobility in the P3HT layers. Layers were characterized "as spin-coated". The difference in magnitude between the channel currents of the two device structures observed in Fig. 4 can be explained by the difference in the $W / L$ ratio of the structures.

Disturbance effects such as modification of the potential in the channel or short circuiting of the semiconductor film due to the channel electrodes in the four probe structure are negligible.

The variation in potential, in dependence on the gate voltage, along the $50 \mu \mathrm{m}$ source-drain channel was monitored via the channel electrodes for a $V_{\mathrm{DS}}$ of $10 \mathrm{~V}$, as shown in Figure 5. The potential in the channel was measured at positions $L_{1}$ and $L_{2}$ via the channel electrodes, shown as $V_{1}$ and $V_{2}$ in the figure. The dashed line shows the extrapolated channel potential from source to drain contact for the case of no contact resistances, given by $V_{1}-V_{2}$, assuming a homogeneous semiconducting channel. The drop in potential due to the contact resistances is given as the difference between the potential at the contacts from the extrapolated channel potential and the actual applied source-drain voltage. This is shown to be substantial in the case of the drain contact (the extracting contact) and slight in the case of the source contact (the injecting contact) leading to a difference of $\sim 5 \mathrm{~V}$ between the applied source-drain voltage and the actual 
potential across the channel. Correcting for the voltage drop due to the contact resistances allowed for the possibility of recalculating a more accurate value for $\mu_{\mathrm{FE}}$, resulting in field effect mobility values in the high $10^{-3} \mathrm{Vs} / \mathrm{cm}^{2}$ range. The values of the contact resistances can then be determined via Ohm's law from the channel current, and were found to be in the order of $\mathrm{k} \Omega$ for the source contact and $\mathrm{k} \Omega$ to $\mathrm{M} \Omega$ for the drain contact for a range of $V_{\mathrm{G}}$ and $V_{\mathrm{DS}}$ values at room temperature.

The symmetry of the device was tested by reversing the polarity, thus switching the source and drain electrodes. The deviation in the results for the voltage drops was found to be negligible in comparison to the magnitude.

It was found that the assumption of a homogeneous semiconducting channel is only valid for currents above $1 \mathrm{nA}$, below which space charge effects are found to lead to unrealistic values for the channel potentials.

[1] H. Bässler, Phys. Status Solidi B 175, 15 (1993).

[2] N. S. Sariciftci, D. Braun, C. Zhang, V. I. Srdanov, A. J. Heeger, G. Stucky, and F. Wudl, Appl. Phys. Lett. 62, 585 (1993)

[3] J. J. Halls, K. Pichler, and R.H. Friend, Appl. Phys. Lett. 68, 3120 (1996).

[4] G. Yu, J. Gao, J.C. Hummelen, F. Wudl, and A. J. Heeger, Science 270, 1789 (1995).

[5] E. von Hauff, J. Parisi, and V. Dyakonov, J. Appl. Phys. 100, 043702 (2006).

[6] P. V. Necliudov, M. S. Shur, D. J. Grundlach, and T. N. Jackson, J. Appl. Phys. 88, 6594 (2000).

[7] R. A. Street and A. Salleo, Appl. Phys. Lett. 81, 2887 (2002).

[8] G. Horowitz, R. Hajlaoui, D. Fichou, and A. E. Kassmi, J. Appl. Phys. 85, 3202 (1999).

[9] B. H. Hamadani and D. Natelson, Appl. Phys. Lett. 84, 443 (2004).

[10] E. von Hauff, V. Dyakonov, and J. Parisi, Sol. Energy Mater. Sol. Cells 87, 149 (2005).

\section{Conclusions}

In this study we employed a four probe geometry to investigate the variation in potential along the sourcedrain channel in P3HT OFETs. This method was used in order to isolate and eliminate the effects of contact resistances which can falsify field effect mobility measurements. It was found that the channel electrodes do not influence the device behaviour at currents $\gg 1 \mathrm{nA}$. The contact resistances were found to be in the order of $\mathrm{k} \Omega$ to $\mathrm{M} \Omega$ for the drain electrode and in the order of $\mathrm{k} \Omega$ for the source electrode. Accounting for the voltage drop across the electrodes led to a correction in the hole field effect mobility from low $10^{-3} \mathrm{Vs} / \mathrm{cm}^{2}$ to high $10^{-3} \mathrm{Vs} / \mathrm{cm}^{2}$ range.

\section{Acknowledgements}

The authors would like to acknowledge Achim Kittel, Leonid Govor, and Martin Knipper for fruitful discussion, and Holger Koch for technical assistance.

[11] S. Narioka, H. Ishii, D. Yoshimura, M. Sei, Y. Ouchi, K. Seki, S. Hasegawa, T. Miyazaki, Y. Harima, and K. Yamashita, Appl. Phys. Lett. 67, 1899 (1995).

[12] T. Shimada, K. Hamaguchi, A. Koma, and F.S. Ohuchi, Appl. Phys. Lett. 72, 1869 (1998).

[13] J. M. Barathan and Y. Yang, J. Appl. Phys. 84, 3207 (1998).

[14] E. J. Meijer, G. H. Gelinck, E. van Veenendaal, V.-H. Huisman, D. M. de Leeuw, and T. M. Klapwijk, Appl. Phys. Lett. 82, 4576 (2003).

[15] B. H. Hamadani and D. Natelson, J. Appl. Phys. 95, 1227 (2004).

[16] L. Bürgi, T. J. Richards, R. H. Friend, and H. Sirringhaus, J. Appl. Phys. 94, 6129 (2003).

[17] P. V. Pesavento, K. P. Puntambeker, C. D. Frisbie, J. C. McKeen, and P. P. Ruden, J. Appl. Phys. 99, 094504 (2006).

[18] B. H. Hamadani and D. Natelson, J. Appl. Phys. 84, 443 (2004). 